Journal of Patient-Centered

Volume 7

Issue 4 -- Women and Cancer

Article 2

$10-23-2020$

\title{
Presence and Distress of Chemotherapy-Induced Peripheral Neuropathy Symptoms in Upper Extremities of Younger and Older Breast Cancer Survivors
}

\author{
Susan Storey \\ Andrea Cohee \\ Diane Von Ah \\ Eric Vachon \\ Noah R. Zanville \\ Patrick O. Monahan \\ Timothy E. Stump \\ Victoria L. Champion
}

Follow this and additional works at: https://aah.org/jpcrr

Part of the Body Regions Commons, Neurology Commons, Oncology Commons, Other Nursing Commons, and the Rehabilitation and Therapy Commons

\section{Recommended Citation}

Storey S, Cohee A, Von Ah D, Vachon E, Zanville NR, Monahan PO, Stump TE, Champion VL. Presence and distress of chemotherapy-induced peripheral neuropathy symptoms in upper extremities of younger and older breast cancer survivors. J Patient Cent Res Rev. 2020;7:295-303. doi: 10.17294/2330-0698.1757

Published quarterly by Midwest-based health system Advocate Aurora Health and indexed in PubMed Central, the Journal of Patient-Centered Research and Reviews (JPCRR) is an open access, peer-reviewed medical journal focused on disseminating scholarly works devoted to improving patient-centered care practices, health outcomes, and the patient experience. 


\title{
Presence and Distress of Chemotherapy-Induced Peripheral Neuropathy Symptoms in Upper Extremities of Younger and Older Breast Cancer Survivors
}

\author{
Susan Storey, PhD, RN, ${ }^{1}$ Andrea Cohee, $\mathrm{PhD}, \mathrm{RN},{ }^{1}$ Diane Von Ah, PhD, RN, ${ }^{1}$ Eric Vachon, PhD, \\ $\mathrm{RN},{ }^{1}$ Noah R. Zanville, PhD, RN, ${ }^{2}$ Patrick O. Monahan, PhD, ${ }^{3,4}$ Timothy E. Stump, MA ${ }^{3}$ Victoria L. \\ Champion, $\mathrm{PhD}, \mathrm{RN}^{1}$ \\ 1Indiana University School of Nursing, Indianapolis, IN; ${ }^{2}$ Miami Cancer Institute, Baptist Health South Florida, \\ Miami, FL; ${ }^{3}$ ndiana University School of Medicine, Indianapolis, IN; ${ }^{4}$ Richard M. Fairbanks School of Public Health, \\ Indianapolis, IN
}
Purpose The purposes of this study were to determine whether the presence of upper extremity chemotherapy- induced peripheral neuropathy (CIPN) symptoms (burning, pins/needles, numbness, pain, and skin crawls) among breast cancer survivors (BCS) varied according to age ( $\leq 45$ years or $55-70$ years) and to examine age group differences in upper extremity CIPN symptom distress.

Methods The study was a secondary analysis of younger $(n=505)$ and older $(n=622)$ BCS. Inclusion criteria were age of $\leq 45$ years or 55-70 years; patient at 3-8 years postdiagnosis; patient received the chemotherapy regimen of paclitaxel, doxorubicin, and cyclophosphamide; and patient did not have recurrence. The Symptom Survivor Checklist was used to assess presence and distress of upper extremity CIPN symptoms. Analyses explored whether age group predicted CIPN symptom presence and distress while controlling for sociodemographic and medical variables.

Results Older BCS reported fewer pins/needles, numbness, and pain symptoms (odds ratios: 0.623-0.751). Heart disease (odds ratios: 1.59-1.70) and progesterone-negative breast cancer (odds ratio: 0.663) were significantly associated with one or more CIPN symptoms. Symptom distress ratings did not differ by age groups; both age groups indicated distress from CIPN symptoms, with $25 \%$ or more reporting distress as "moderately" or "quite a bit."

Conclusions Younger BCS reported more upper extremity CIPN symptoms. BCS in both groups continued to report bothersome CIPN symptoms years after treatment. Study findings will assist clinicians in identifying BCS at higher risk for upper extremity CIPN as well as inform development of appropriate tailored interventions to mitigate these symptoms and facilitate restoration to age-related baseline function, thereby improving quality of life for BCS. (J Patient Cent Res Rev. 2020;7:295-303.)

Keywords breast cancer; survivorship; chemotherapy-induced peripheral neuropathy; upper extremity; symptom bother; symptom distress

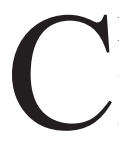
hemotherapy-induced peripheral neuropathy (CIPN) is one of the most prevalent, persistent, and disruptive symptoms associated with common antineoplastic treatments (eg, taxanes) for breast cancer. ${ }^{1-5}$

Corresponding author: Susan Storey, $\mathrm{PhD}, \mathrm{RN}$, Department of Community Health Systems, Indiana University School of Nursing, 600 Barnhill Drive, Indianapolis, IN 46202 (sustorey@iu.edu)
Approximately two-thirds of breast cancer survivors (BCS) report symptoms of CIPN during cancer treatment, ${ }^{6}$ with as many as $42 \%$ reporting lingering symptoms for up to 3 years posttreatment. ${ }^{2,7-10}$ Symptoms of CIPN include sensations of burning, pins/needles, numbness, ${ }^{1,5,9}$ pain, $, 1,5$ and/or skin crawls affecting the hands and/or feet. ${ }^{1,4,5,11-13}$ Although treatments have improved, the continued reliance on taxanes (eg, paclitaxel) and other neurotoxic agents to treat breast cancer has contributed to serious and, at times, permanent changes that significantly interfere with survivors' daily functioning. $5,7,9,11,12$ 
The onset of CIPN in BCS has been associated with sociodemographic $\left(\right.$ age $^{7,11,14,15}$ race, ${ }^{2,9,15}$ income, ${ }^{16}$ education, ${ }^{16}$ and alcohol consumption ${ }^{16}$ ) and medical characteristics (comorbidities, ${ }^{5,11,16}$ hormone status, ${ }^{2}$ tumor size, ${ }^{10}$ body mass index, ${ }^{2,9,15}$ number of positive nodes, ${ }^{10}$ and years from diagnosis ${ }^{17,18}$ ). Age in particular may be an important risk factor in both CIPN symptom presence and distress. ${ }^{7,11,14,15}$ While several studies have found a higher prevalence and longer duration of CIPN symptoms in older BCS ( $\geq 60$ years of age), ,7,11,14-16 others have found a higher prevalence of CIPN symptoms in younger BCS.,19 Further still, some studies found no association between age and the presence of CIPN symptoms. ${ }^{20,21}$ Most of these studies have concentrated exclusively on the lower extremities, with little focus on upper extremity CIPN symptoms.

Determining the impact of upper extremity CIPN symptoms is important to study, as fine motor movements can be impaired, ${ }^{22}$ potentially interfering with the ability of BCS to perform daily activities. Understanding age-related differences in the presence and impact of upper extremity (shoulders, arms, hands, fingers) CIPN symptoms is also important given that symptoms may differentially affect independence and quality of life. ${ }^{5,17,19}$ While lower extremity CIPN may be more distressing for older BCS (eg, concerns about increased falls), upper extremity CIPN may be more distressing for younger BCS (eg, concerns about managing work and family responsibilities).

This study examined upper extremity CIPN symptoms among BCS according to age group (younger vs older BCS). Its aims were twofold. Aim 1 sought to determine whether the presence of upper extremity CIPN symptoms (burning, pins/needles, numbness, pain, and skin crawls) varied according to age group. Aim 2 sought to examine age group differences in level of distress from upper extremity CIPN symptoms. This information would be particularly useful for clinicians and researchers challenged in the management of long-term and troubling CIPN symptoms. Significant findings could assist with identifying BCS at higher risk for CIPN and inform the development of tailored interventions to mitigate these symptoms.

\section{METHODS}

\section{Study Design and Sample}

This study is a secondary analysis of 1127 BCS using cohort data from an earlier cross-sectional quality-oflife study that included several self-reported measures for common symptoms experienced by BCS. Details of the parent study were described comprehensively in a 2014 publication. ${ }^{23}$ Briefly, BCS were eligible if they: 1) were 45 years old or younger (younger BCS) or 55-70 years old (older BCS) at diagnosis; 2) were 3-8 years postdiagnosis without recurrence; and 3) received a chemotherapy regimen that included paclitaxel, doxorubicin, and cyclophosphamide as part of their initial breast cancer treatment. BCS who were 46-54 years of age were excluded to avoid the potentially confounding perimenopausal timeframe.

The secondary analysis presented hereafter was approved by the coordinating university's institutional review board in 2019.

\section{Measures}

Sociodemographic Variables (Independent Variables): Sociodemographic variables, including age, race, income, education, and alcohol consumption, were self-reported. Age was coded as a dichotomous variable of younger $(\leq 45$ years) or older (55-70 years), which was consistent with the targeted enrollment groups as it is less common for BCS over 70 years of age to receive this treatment regimen. Race was coded as a categorical variable (white or nonwhite), income was coded as a dichotomous variable $(<\$ 75,000$ or $\geq \$ 75,000$ ), and education was coded as a continuous variable for years of education. Alcohol consumption was queried by asking, "On those days that you drink alcoholic beverages, about how many do you usually have?" Response options were $>5$ drinks, 3-5 drinks, 1-2 drinks, and I do not drink (none). For this study, the alcohol consumption variable was recoded into categories of $\geq 3$ drinks per day, 1-2 drinks per day, or none.

Medical Variables (Independent Variables): Medical information was collected via self-report from BCS and included comorbidities (heart disease, hypertension, diabetes), hormone status, tumor size, body mass index (calculated from height and weight), number of positive nodes, and years from diagnosis. Researchers reviewed medical records to verify patient-reported information.

CIPN Symptoms (Dependent Variables): The Symptom Survivor Checklist was developed for this study based on review of CIPN literature..$^{1,4,5,11-13}$ The checklist is a 12-item scale that assesses 5 common upper extremity CIPN symptoms on the affected side, including burning, pins/needles, numbness, pain, and skin crawls. BCS were asked if the symptoms were present (yes/no). If present, then BCS rated the extent to which they were bothered or distressed by symptoms on a 5 -point ordinal scale $(0$ $=$ not at all; $1=$ slightly; $2=$ moderately; $3=$ quite a bit; 4 = extremely). Higher scores were indicative of greater symptom distress. Cronbach's alpha for this scale in our study was 0.78 .

Data Analysis: Data analysis was conducted using SPSS Statistics 25.0 (IBM Corp.), and significance was set at 
$\mathrm{P}<0.05$. Two-tailed tests were used for all comparisons. Prior to the analysis, Shapiro-Wilks tests were used to test for normality of the data. Descriptive statistics were used to summarize the distributions of sociodemographic and medical variables. To examine age group differences in sociodemographic and medical characteristics, we used Pearson's chi-squared tests for categorical variables (race, income, marital status, comorbidities, hormone status, alcohol consumption) and independent $t$-tests for continuous variables (years of education, body mass index, years from diagnosis, number of positive nodes, tumor size).

For study aim 1, we first conducted 5 separate Pearson's chi-squared tests to determine if there was a significant association between age group and the presence of each of the 5 upper extremity CIPN symptoms: burning, pins/needles, numbness, pain, and skin crawls. Three significant symptoms were analyzed in a series of separate binary logistic regression analyses to determine whether age group was associated with the presence of the symptoms while controlling for sociodemographic (age, race, income, education, alcohol consumption) and medical (heart disease, hypertension, diabetes, hormone status, tumor size, body mass index, number of positive nodes, years from diagnosis) variables that theoretically could influence CIPN. In the regression model, each symptom was entered as a dependent variable; age was an independent variable along with other sociodemographic and medical covariates. Using a backward deletion approach, statistically nonsignificant sociodemographic and medical characteristics were removed until the final model was achieved.

For study aim 2, Mann-Whitney U tests were conducted to examine age group differences in symptom distress. Participants who did not report any CIPN symptoms were removed from the Mann-Whitney $U$ analysis. If significant, backward deletion ordinal logistic regression analyses would have been conducted to determine if age group predicted symptom distress while controlling for sociodemographic and medical characteristics.

\section{RESULTS}

\section{Sample Characteristics}

Sociodemographic and medical characteristics of younger $(n=505)$ and older $(n=622)$ BCS are shown in Table 1. On average, BCS were 6 years posttreatment. Compared to older BCS, younger BCS had significantly higher incomes, were more likely to be married, had fewer comorbidities, consumed more alcohol, and had more years of education.

\section{Aim 1. Presence of CIPN Symptoms}

Table 2 shows the chi-squared results for the presence of upper extremity CIPN symptoms for the total sample and by age group. Younger age group was significantly associated with presence of 3 CIPN symptoms: pins/needles $(\mathrm{P}=0.021)$, numbness $(\mathrm{P}<0.001)$, and pain $(\mathrm{P}<0.001)$.

Results of logistic regression analyses are summarized in Table 3. Age significantly predicted the presence of pins/ needles, numbness, and pain symptoms. Older BCS had lower odds of reporting upper extremity pins/needles, numbness, and pain symptoms compared to younger BCS (odds ratios: 0.623-0.751). Comorbid heart disease also was a significant predictor of these 3 upper extremity CIPN symptoms. BCS with heart disease were 1.59-1.70 times more likely to report pins/needles, numbness, and pain symptoms. Additionally, hormone status predicted the presence of the pins/needles symptom. Those with positive progesterone receptor (PR) status had lower odds (odds ratio: 0.663 ) of experiencing the pins/needles symptom. While statistically significant, the variation (R2) in the dependent variable based on our models ranged from $2.3 \%$ to $2.9 \%$, demonstrating a small but consistent relationship between age and upper extremity CIPN symptoms (pins/needles, numbness, pain).

\section{Aim 2. Symptom Distress}

Table 4 shows the level of distress associated with CIPN symptoms by age group. Distress scores ranged from 1 (slightly) to 3 (quite a bit), with the typical score (ie, median) indicating BCS were slightly or moderately distressed. It should be noted that 2 or 3 , depending on the symptom, was not only the maximum score reported but also the 75 th percentile, indicating that $25 \%$ of BCS perceived "moderately" or "quite a bit" of distress for each symptom. Mann-Whitney U tests showed no significant age group differences in upper extremity CIPN symptom distress. Given that there were no statistically significant age group differences in symptom distress scores, ordinal logistic regression was not conducted.

\section{DISCUSSION}

The purpose of this study was to describe the presence and distress level of upper extremity CIPN symptoms in BCS 3 or more years posttreatment. The findings offer several key insights. First, our results add to the literature showing that younger BCS report the presence of upper extremity CIPN symptoms more often than older BCS. Second, comorbid heart disease and hormone status were identified as risk factors for upper extremity CIPN symptoms. Third, both younger and older BCS continued to report upper extremity CIPN symptoms as distressing several years posttreatment. 
Table 1. Comparison of Sociodemographic and Medical Characteristics of Younger ( $\leq 45$ Years) and Older (55-70 Years) Breast Cancer Survivors

\begin{tabular}{|c|c|c|c|}
\hline Variables & 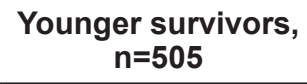 & $\begin{array}{c}\text { Older survivors, } \\
n=622\end{array}$ & $P$ \\
\hline \multicolumn{4}{|l|}{ Race, n (\%) } \\
\hline White & $459(91)$ & $582(95)$ & 0.114 \\
\hline Non-White & $46(9)$ & $30(5)$ & \\
\hline \multicolumn{4}{|l|}{ Income, n (\%) } \\
\hline$<\$ 75,000$ & $224(45)$ & $427(72)$ & $<0.001$ \\
\hline$\geq \$ 75,000$ & $271(55)$ & $167(28)$ & \\
\hline \multicolumn{4}{|l|}{ Marital status, n (\%) } \\
\hline Married & $417(83)$ & $419(69)$ & $<0.001$ \\
\hline Non-Married & $85(17)$ & $188(31)$ & \\
\hline Missing & $3(0.6)$ & $15(2)$ & \\
\hline \multicolumn{4}{|l|}{ Comorbidities, n (\%) } \\
\hline Heart disease: Yes & $36(7)$ & $83(13)$ & $<0.001$ \\
\hline Hypertension: Yes & $74(15)$ & $328(53)$ & $<0.001$ \\
\hline Diabetes: Yes & $28(6)$ & $93(15)$ & $<0.001$ \\
\hline \multicolumn{4}{|l|}{ Positive hormone status, n (\%) } \\
\hline Estrogen: Yes & $334(67)$ & $452(75)$ & 0.011 \\
\hline Progesterone: Yes & $298(62)$ & $383(65)$ & 0.408 \\
\hline HER2/neu: Yes & $111(27)$ & $129(25)$ & 0.548 \\
\hline \multicolumn{4}{|l|}{ Alcohol consumption, n (\%) } \\
\hline 0 drinks & $144(29)$ & $251(40)$ & $<0.001$ \\
\hline $1-2$ drinks & $294(58)$ & $347(56)$ & \\
\hline 3 or more & $67(13)$ & $24(4)$ & \\
\hline Years of education, mean (SD) & $14.8(2.6)$ & $14.1(2.7)$ & $<0.001$ \\
\hline Body mass index, mean (SD) & $27.8(6.1)$ & $28.4(5.9)$ & 0.102 \\
\hline Years from diagnosis, mean (SD) & $5.9(1.5)$ & $6(1.5)$ & 0.336 \\
\hline Number of positive nodes, mean (SD) & $1.5(2.6)$ & $1.7(3.2)$ & 0.272 \\
\hline Tumor size in $\mathrm{cm}$, mean (SD) & $2(1.4)$ & $1.9(1.3)$ & 0.394 \\
\hline
\end{tabular}

HER2, human epidermal growth factor receptor 2; SD, standard deviation.

Table 2. Presence of Peripheral Neuropathy Symptoms by Total Sample and Between Younger ( $\leq 45$ Years) and Older (55-70 Years) Breast Cancer Survivors

\begin{tabular}{lcccc}
\hline Symptom & $\begin{array}{c}\text { All, } \\
\mathbf{N = 1 1 2 7}\end{array}$ & $\begin{array}{c}\text { Younger survivors, } \\
\mathbf{n = 5 0 5}\end{array}$ & $\begin{array}{c}\text { Older survivors, } \\
\mathbf{n = 6 2 2}\end{array}$ & $\boldsymbol{P}^{*}$ \\
\hline Burning, n (\%) & $119(9)$ & $60(12)$ & $59(10)$ & 0.284 \\
Pins/needles, n (\%) & $368(33)$ & $185(37)$ & $183(30)$ & 0.021 \\
Numbness, n (\%) & $442(39)$ & $232(46)$ & $210(34)$ & $<0.001$ \\
Skin crawls, n (\%) & $109(10)$ & $58(11)$ & $51(8)$ & 0.085 \\
Pain, n (\%) & $347(31)$ & $189(37)$ & $158(25)$ & $<0.001$ \\
\hline
\end{tabular}

*Determined by chi-squared test. 
Table 3. Predictors of Symptom Presence (Yes/No) Among BCS Based on Logistic Regression Analysis

\begin{tabular}{|c|c|c|c|c|c|}
\hline Variable & Standard beta & SE beta & $P$ & Odds ratio & $95 \% \mathrm{Cl}$ \\
\hline Older vs younger BCS & -0.286 & 0.144 & 0.048 & 0.751 & $0.566,0.997$ \\
\hline Heart disease: present vs not present & 0.457 & 0.224 & 0.041 & 1.59 & $1.019,2.451$ \\
\hline PR hormone status: positive vs negative & -0.412 & 0.163 & 0.005 & 0.663 & $0.495,0.886$ \\
\hline \multicolumn{6}{|c|}{ Numbness $\left[n=442 ; X^{2}=5.056, d f=4 ; R^{2}=0.023, P=0.001\right]$} \\
\hline Variable & Standard beta & SE beta & $\boldsymbol{P}$ & Odds ratio & $95 \% \mathrm{Cl}$ \\
\hline Older vs younger BCS & -0.449 & 0.137 & 0.001 & 0.638 & $0.488,0.835$ \\
\hline Heart disease: present vs not present & 0.466 & 0.218 & 0.032 & 1.59 & $1.04,2.44$ \\
\hline \multicolumn{6}{|c|}{ Pain $\left[n=347 ; X^{2}=3.17, d f=3 ; R^{2}=0.025 ; P<0.001\right]$} \\
\hline Variable & Standard beta & SE beta & $\boldsymbol{P}$ & Odds ratio & $95 \% \mathrm{CI}$ \\
\hline Older vs younger BCS & -0.474 & 0.145 & 0.001 & 0.623 & $0.469,0.827$ \\
\hline Heart disease: present vs not present & 0.551 & 0.223 & 0.013 & 1.70 & $1.12,2.68$ \\
\hline
\end{tabular}

BCS, breast cancer survivors; df, degrees of freedom; PR, progesterone receptor; SE, standard error.

Table 4. Comparison of Symptom Distress by Age Groups

\begin{tabular}{|c|c|c|c|c|}
\hline Symptom & $\begin{array}{c}\text { Score range, } \\
\text { potential }^{\mathrm{a}} \text { (actual) }\end{array}$ & $\begin{array}{l}\text { Younger survivors, } \\
\text { median (P25, P75) }\end{array}$ & $\begin{array}{l}\text { Older survivors, } \\
\text { median (P25, P75) }\end{array}$ & $P^{b}$ \\
\hline Burning [n=290] & $0-4(1-3)$ & $\begin{array}{c}2.0(1,2) \\
n=120\end{array}$ & $\begin{array}{c}2.0(1,3) \\
n=170\end{array}$ & 0.521 \\
\hline Pins/needles [n=347] & $0-4(1-2)$ & $\begin{array}{c}1.0(1,2) \\
n=173\end{array}$ & $\begin{array}{c}1.0(1,2) \\
n=174\end{array}$ & 0.992 \\
\hline Numbness [n=376] & $0-4(1-3)$ & $\begin{array}{c}2.0(1,3) \\
n=193\end{array}$ & $\begin{array}{c}2.0(1,2) \\
n=183\end{array}$ & 0.117 \\
\hline Pain $[n=332]$ & $0-4(1-2)$ & $\begin{array}{c}1.0(1,2) \\
n=181\end{array}$ & $\begin{array}{c}1.0(1,2) \\
n=151\end{array}$ & 0.330 \\
\hline Skin crawls [n=107] & $0-4(1-3)$ & $\begin{array}{c}2.0(1,2) \\
n=56\end{array}$ & $\begin{array}{c}2.0(1,3) \\
n=51\end{array}$ & 0.885 \\
\hline
\end{tabular}

a Survivors rated extent of distress on a 5-point scale: 0 = not at all; 1 = slightly; 2 = moderately; $3=$ quite a bit; 4 = extremely).

${ }^{b}$ Determined by Mann-Whitney $U$ test.

P25, 25th percentile; $P 75,75$ th percentile.

\section{Aim 1. Presence of CIPN Symptoms}

While factors such as treatment duration and the intensity of taxane exposure that BCS receive during treatment are known factors for developing chronic CIPN, ${ }^{10-12}$ the role of age on these symptoms is less clear. In our study, we found younger BCS were more likely to report upper extremity CIPN symptoms of pins/needles, numbness, and pain compared to their older counterparts. This finding is consistent with other studies that have shown younger BCS may be at higher risk of developing CIPN symptoms. ${ }^{2,19} \mathrm{Ali}$ and colleagues found younger BCS had a higher risk of CIPN than older BCS. However, the researchers did not discuss how CIPN was defined by location, limiting the ability to generalize the finding to other BCS populations. ${ }^{2}$ Although underlying mechanisms contributing to age-related differences in CIPN symptoms are not clear, one possible explanation is that, unlike younger BCS, older BCS may have preexisting neuropathy and/or other neurodegenerative loss from comorbid conditions that lessen their perception of these symptoms. 
In contrast to our findings, other studies have shown that older BCS are at increased risk for CIPN symptoms. Hershman and colleagues linked clinical trial data with Medicare claims and found older age to be the only demographic factor associated with the presence of upper and lower extremity CIPN symptoms. ${ }^{13}$ However, the mean age of the sample (73 years) was older, which may have influenced the study findings. Additionally, the lack of access to baseline neurologic status - to determine if symptoms of neuropathy were preexisting or new-onset - may have resulted in higher reporting of symptom presence.

Similarly, Lichtman and colleagues examined neurotoxicity (neurosensory, neuromotor, neurocortical, or neurocerebellar) symptoms among BCS $(\mathrm{N}=1048)$ in 3 age categories: $<55$ years, $55-64$ years, and $\geq 65$ years. ${ }^{24}$ Compared to those in the younger age categories, women who were $\geq 65$ years of age $(n=272)$ reported earlier onset and the presence of neurotoxicity with first- and secondline chemotherapy treatments. ${ }^{24}$ However, the focus of the study was specific to neurotoxicity, which may influence neuropathy but cannot be directly extrapolated to reflect CIPN symptoms.

Other researchers have found no association between age and CIPN symptoms. Schneider and colleagues studied BCS $(\mathrm{N}=4554)$ in 3 age categories $(\leq 45,46-65$, and $>65$ years) all receiving adjuvant taxane therapy. ${ }^{25}$ Although they found CIPN symptoms in upper and lower extremities were common complications of treatment, age was not an associated factor. ${ }^{25}$ However, the researchers indicated that a lack of uniform and validated CIPN assessments may have decreased the reliability of the study findings. More research is needed to assess if upper extremity CIPN symptoms differ by age among BCS.

Although diabetes has been noted as a risk factor for CIPN in BCS, ${ }^{13}$ we did not find diabetes to be associated with upper extremity CIPN in our study. This finding may be related to the typical presentation of CIPN symptoms in the lower extremities in people with diabetes, ${ }^{26}$ whereas our study focused on upper extremity symptoms. Additionally, the number of BCS with comorbid diabetes $(\mathrm{n}=121)$ in our study was low.

An important finding from our study was that BCS with comorbid heart disease were more likely to report the presence of pins/needles, numbness, and pain symptoms in the years following treatment. Heart disease, hypertension, and diabetes, the most frequently documented comorbidities in BCS, ${ }^{27-33}$ are all associated with an increased risk of CIPN. ${ }^{6,30,31}$ The BCS in our study received the same therapeutic regimen, which included two of the most common cardiotoxic chemotherapies (doxorubicin and paclitaxel). Because we examined heart disease broadly, we were not able to identify specific cardiac pathologies nor determine if they occurred prior to or after cancer diagnosis and treatment, limiting our ability to determine if cardiovascular disease is a direct risk factor for CIPN, especially in the upper extremities. The influence of comorbid heart disease on CIPN symptoms among BCS has not been well studied. As $\mathrm{BCS}$ are living longer, the role of comorbid conditions on CIPN symptoms is important to understand and warrants further research. Clinicians should assess for the presence of CIPN symptoms in BCS with a history of heart disease.

Finally, although estrogen receptor status did not predict upper extremity CIPN symptoms, progesterone status did. Specifically, BCS who were PR-positive were less likely to report sensations of pins/needles in the years following treatment than BCS who were PRnegative. Our findings are consistent with another study that reported PR-negative status was associated with an increased risk of developing CIPN. ${ }^{9}$ Studies have suggested that progesterone may have neuroprotective effects on the central and peripheral nervous systems by promoting repair of myelin. ${ }^{33,34}$ Although, the degree to which this may reduce the severity of taxane-induced CIPN is unclear. More research is needed to understand the role of hormone status on CIPN symptoms.

\section{Aim 2. Symptom Distress}

Results of our study found that, within an average of 6 years following treatment, BCS reported being distressed by CIPN symptoms. Although younger BCS were more likely than older BCS to report upper extremity CIPN symptoms, we found no difference in distress levels among younger and older BCS.

Studies examining differences by age of BCS on symptom distress from upper extremity CIPN are lacking. We found two studies that assessed CIPN symptom distress by age. Tanabe and colleagues found CIPN symptoms were more severe and persistent among older BCS ( $\geq 60$ years of age) ${ }^{14}$ However, the authors assessed CIPN symptoms in combination of upper and lower extremities and did not differentiate symptom distress by location. Wong and colleagues assessed age-related differences in both upper and lower extremity CIPN symptoms in cancer survivors $(n=425)$ and found younger survivors reported more severe pain and more interference in activity from CIPN symptoms when compared to older survivors. ${ }^{19}$ However, the study included heterogenous cancer diagnoses as well as survivors with metastatic disease, which may have influenced the findings. Other studies have shown younger survivors with CIPN report more difficulty with 
general activities, work, and other components of quality of life, ${ }^{19,35,36}$ an important finding given that they have not experienced nor adapted to the age-related losses that commonly occur with aging. ${ }^{19}$

The lack of association between age and CIPN distress observed in our study may have been a result of our choice of measure, which only assessed upper extremity CIPN symptoms. Future research should assess the symptom experience of upper and lower CIPN symptoms individually as well as collectively. Assessing upper and lower extremity function separately in BCS may provide helpful information as it relates to the differential impact of CIPN on function and daily activities.

It is important to note, although we did not observe an age-specific difference in the level of distress from upper extremity CIPN symptoms, both younger and older BCS still indicated distress from these symptoms several years posttreatment, with $25 \%$ or more reporting "moderately" or "quite a bit" of distress depending on the particular symptom. This finding supports other research that illustrates the lingering long-term impact of CIPN symptoms experienced by BCS. Future research should consider examining upper extremity CIPN symptoms in younger and older BCS longitudinally across the trajectory of cancer treatment into survivorship.

This study had several strengths and contributes to a growing body of literature by identifying age-related differences in 3 upper extremity CIPN symptoms. To our knowledge, this is one of the first studies to evaluate the impact of age and medical variables on specific upper extremity CIPN symptoms in BCS and in a patient cohort that is $>3$ years posttreatment. Results from our analysis were drawn from a large nationally representative sample of $\mathrm{BCS}$ who received one of the most common taxane-based regimens (a primary driver of CIPN in BCS), increasing the generalizability of findings. The study included a 10year separation between younger and older BCS, reducing variation in the sample. Given our findings that younger $\mathrm{BCS}$ are at risk for experiencing CIPN symptoms, future research should explore the development and testing of age-appropriate interventions to restore function, reduce distress, and improve quality of life.

\section{Limitations}

Study findings also need to be considered in the context of several limitations. First, because of its cross-sectional design, neurological diagnostic testing was not available, limiting the ability to identify baseline CIPN symptoms or determine if the symptoms evolved over time. Second, the upper age limit of older BCS in our study was 70 years, whereas other studies included much older subjects (ie, $\geq 65$ years), which may have influenced these findings. Third, we did not have access to information on cumulative exposure to taxanes for participants, which could have an impact on CIPN symptoms. Fourth, because African American women, who have a 2- to 3-fold higher risk of taxane-induced CIPN than Caucasian women, ${ }^{11,13}$ were not well represented in this study, generalizability to all race/ethnicities is limited. Finally, although the Survivor Symptom Checklist demonstrated an acceptable Cronbach's alpha, additional validation of the tool is warranted. Despite these limitations, this study's findings add to the body of knowledge as it relates to presence and distress of upper extremity CIPN symptoms from the perspectives of younger and older BCS, an understudied area.

\section{Implications for Practice}

The information from this study will be particularly useful for clinicians and researchers challenged in the care of long-term and troubling CIPN symptoms. We found upper extremity CIPN symptoms can be distressing for BCS years posttreatment. CIPN symptoms are typically assessed in combination with lower extremity symptoms at the onset and intermittently during active treatment for cancer. Our findings elucidate the need for ongoing assessment and monitoring of upper extremity CIPN symptoms in BCS throughout the cancer trajectory, well into survivorship. These assessments could facilitate referrals to rehabilitative programs to implement agespecific rehabilitative interventions geared towards maximizing safety and physical function, hopefully improving overall quality of life for BCS.

Future research should consider examining upper extremity CIPN symptoms longitudinally in a more diverse sample of BCS across the trajectory of cancer treatment and into survivorship. Additionally, research testing age-appropriate tailored interventions toward mitigating these symptoms in BCS may facilitate restoration to age-related baseline functional status.

\section{CONCLUSIONS}

Findings from this study have integral implications for occupational and rehabilitative staff when developing treatment plans aimed toward improving safety and functional performance for long-term BCS in survivorship programs. Of particular importance is the finding that younger BCS, for whom work is likely to be a critical issue, are more likely than older BCS to report pain, tingling, and numbness in their upper extremities. Although research specific to upper extremity CIPN symptoms in BCS is nominal, several studies have implicated the presence, number, type, and duration of CIPN symptoms (upper/lower combined) with difficulty performing work-related tasks. ${ }^{36-38}$ Successful 
rehabilitation efforts would facilitate strategies to increase participation in work, spur earlier return to work, and improve activities of daily living and overall quality of life.

\section{Patient-Friendly Recap}

- Survivors of breast cancer may experience pain, numbness, or other sensations in their extremities years after treatment. These symptoms stem from a nerve condition called chemotherapy-induced peripheral neuropathy (CIPN).

- The authors studied if presence and degree of distress from CIPN symptoms varied by age between breast cancer survivors diagnosed at a younger ( $\leq 45$ years) or older (55-70 years) age.

- They found that upper extremity pain, pins/needles, and numbness were more frequently reported by younger survivors. They also significantly associated heart disease and progesterone-negative breast cancer with certain CIPN symptoms.

- Both age groups largely indicated that CIPN symptoms caused them a slight-to-moderate degree of distress years after treatment.

\section{Author Contributions}

Study design: Storey, Cohee, Zanville, Champion. Data acquisition or analysis: Storey, Monahan, Stump, Champion. Manuscript drafting: Storey, Cohee, Von Ah, Vachon, Zanville, Champion. Critical revision: Von Ah.

\section{Conflicts of Interest}

None.

\section{Funding Sources}

This study was coordinated by the ECOG-ACRIN Cancer Research Group (co-chairs: Peter J. O’Dwyer, Mitchell D. Schnall) and supported by the National Cancer Institute of the National Institutes of Health (NIH) under the award numbers CA189828 and CA180795. The content is solely the responsibility of the authors and does not necessarily represent the official views of the NIH, nor does mention of trade names, commercial products, or organizations imply endorsement by the U.S. Government. Dr. Storey's work is funded in part through a Release Time for Research award from the IUPUI Office of the Vice Chancellor for Research, the Research Investment Fund from the Indiana University School of Nursing, and an RE01 grant from the Oncology Nursing Foundation. Dr. Cohee is supported by Indiana Clinical and Translational Sciences Institute's KL2 Program (UL1TR002529, May 18, 2018-April 30, 2023). Prior research reported in this publication was supported by the National Cancer Institute under award number K05CA175048. Its content is solely the responsibility of the authors and does not necessarily represent the official views of the NIH, including the National Cancer Institute or the National Institute of Nursing Research.

\section{References}

1. Miltenburg NC, Boogerd W. Chemotherapy-induced neuropathy: a comprehensive survey. Cancer Treat Rev. 2014;40:872-82. Crossref

2. Mustafa Ali M, Moeller M, Rybicki L, Moore HC. Long-term peripheral neuropathy symptoms in breast cancer survivors. Breast Cancer Res Treat. 2017;166:519-26. Crossref

3. Majithia N, Temkin SM, Ruddy KJ, Beutler AS, Hershman DL, Loprinzi CL. National Cancer Institute-supported chemotherapy-induced peripheral neuropathy trials: outcomes and lessons. Support Care Cancer. 2016;24:1439-47. Crossref

4. Jang CE, Jung MS, Sohn EH, et al. The evaluation of changes in peripheral neuropathy and quality-of-life using low-frequency electrostimulation in patients treated with chemotherapy for breast cancer: a study protocol. Trials. 2018;19(1):526. Crossref

5. Kırca K, Kutlutürkan S. Symptoms experience and quality of life in the patients with breast cancer receiving the taxane class of drugs. Eur J Breast Health. 2018;14:148-55. Crossref

6. Coffeen U, Sotomayor-Sobrino MA, Jiménez-González A, et al. Chemotherapy-induced neuropathic pain characteristics in Mexico's National Cancer Center pain clinic. J Pain Res. 2019;12:1331-9. Crossref

7. Eckhoff L, Knoop A, Jensen MB, Ewertz M. Persistence of docetaxel-induced neuropathy and impact on quality of life among breast cancer survivors. Eur J Cancer. 2015;51:292300. $\underline{\text { Crossref }}$

8. Seretny M, Currie GL, Sena ES, et al. Incidence, prevalence, and predictors of chemotherapy-induced peripheral neuropathy: a systematic review and meta-analysis. Pain. 2014;155:2461-70. Crossref

9. Bao T, Basal C, Seluzicki C, Li SQ, Seidman AD, Mao JJ. Long-term chemotherapy-induced peripheral neuropathy among breast cancer survivors: prevalence, risk factors, and fall risk. Breast Cancer Res Treat. 2016;159:327-33. Crossref

10. Bandos H, Melnikow J, Rivera DR, et al. Long-term peripheral neuropathy in breast cancer patients treated with adjuvant chemotherapy: NRG Oncology/NSABP B-30. J Natl Cancer Inst. 2017;110(2):djx162. Crossref

11. Bhatnagar B, Gilmore S, Goloubeva O, et al. Chemotherapy dose reduction due to chemotherapy induced peripheral neuropathy in breast cancer patients receiving chemotherapy in the neoadjuvant or adjuvant settings: a single-center experience. Springerplus. 2014;3:366. Crossref

12. Ewertz M, Qvortrup C, Eckhoff L. Chemotherapy-induced peripheral neuropathy in patients treated with taxanes and platinum derivatives. Acta Oncol. 2015;54:587-91. Crossref

13. Hershman DL, Lacchetti C, Dworkin RH, et al. Prevention and management of chemotherapy-induced peripheral neuropathy in survivors of adult cancers: American Society of Clinical Oncology clinical practice guideline. J Clin Oncol. 2014;32:1941-67. Crossref

14. Tanabe Y, Hashimoto K, Shimizu C, et al. Paclitaxelinduced peripheral neuropathy in patients receiving adjuvant chemotherapy for breast cancer. Int J Clin Oncol. 2013;18:132-8. Crossref

15. Greenlee H, Hershman DL, Shi Z, et al. BMI, lifestyle factors and taxane-induced neuropathy in breast cancer patients: the Pathways Study. J Natl Cancer Inst. 2016;109(2):djw206. Crossref 
16. Hershman DL, Till C, Wright JD, et al. Comorbidities and risk of chemotherapy-induced peripheral neuropathy among participants 65 years or older in Southwest Oncology Group clinical trials. J Clin Oncol. 2016;34:3014-22. Crossref

17. Molassiotis A, Cheng HL, Lopez V, et al. Are we misestimating chemotherapy-induced peripheral neuropathy? Analysis of assessment methodologies from a prospective, multinational, longitudinal cohort study of patients receiving neurotoxic chemotherapy. BMC Cancer. 2019;19(1):132. Crossref

18. Staff NP, Grisold A, Grisold W, Windebank AJ. Chemotherapyinduced peripheral neuropathy: a current review. Ann Neurol. 2017;81:772-81. Crossref

19. Wong ML, Cooper BA, Paul SM, et al. Age-related differences in patient-reported and objective measures of chemotherapyinduced peripheral neuropathy among cancer survivors. Support Care Cancer. 2019;27:3905-12. Crossref

20. Nurgalieva Z, Xia R, Liu CC, Burau K, Hardy D, Du XL. Risk of chemotherapy-induced peripheral neuropathy in large population-based cohorts of elderly patients with breast, ovarian, and lung cancer. Am J Ther. 2010;17:148-58. $\underline{\text { Crossref }}$

21. Wolf SL, Barton DL, Qin R, et al. The relationship between numbness, tingling, and shooting/burning pain in patients with chemotherapy-induced peripheral neuropathy (CIPN) as measured by the EORTC QLQ-CIPN20 instrument, N06CA. Support Care Cancer. 2012;20:625-32. Crossref

22. Starobova H, Vetter I. Pathophysiology of chemotherapyinduced peripheral neuropathy. Front Mol Neurosci. 2017;10:174. Crossref

23. Champion VL, Wagner LI, Monahan PO, et al. Comparison of younger and older breast cancer survivors and age-matched controls on specific and overall quality of life domains. Cancer. 2014;120:2237-46. Crossref

24. Lichtman SM, Hurria A, Cirrincione CT, et al. Paclitaxel efficacy and toxicity in older women with metastatic breast cancer: combined analysis of CALGB 9342 and 9840. Ann Oncol. 2011;23:632-8. Crossref

25. Schneider BP, Zhao F, Wang M, et al. Neuropathy is not associated with clinical outcomes in patients receiving adjuvant taxane-containing therapy for operable breast cancer. J Clin Oncol. 2012;30:3051-7. Crossref

26. Hicks CW, Selvin E. Epidemiology of peripheral neuropathy and lower extremity disease in diabetes. Curr Diab Rep. 2019;19(10):86. Crossref

27. Sharma N, Narayan S, Sharma R, Kapoor A, Kumar N, Nirban R. Association of comorbidities with breast cancer: an observational study. Trop J Med Res. 2016;19:168-71.
28. Edwards BK, Noone AM, Mariotto AB, et al. Annual report to the Nation on the status of cancer, 1975-2010, featuring prevalence of comorbidity and impact on survival among persons with lung, colorectal, breast, or prostate cancer. Cancer. 2014;120:1290-314. Crossref

29. Ewertz M, Land LH, Dalton SO, Cronin-Fenton D, Jensen MB. Influence of specific comorbidities on survival after early-stage breast cancer. Acta Oncol. 2018;57:129-34. Crossref

30. Fu MR, Axelrod D, Guth AA, et al. Comorbidities and quality of life among breast cancer survivors: a prospective study. J Pers Med. 2015;5:229-42. Crossref

31. Kus T, Aktas G, Kalender ME, et al. Taxane-induced peripheral sensorial neuropathy in cancer patients is associated with duration of diabetes mellitus: a single-center retrospective study. Support Care Cancer. 2016;24:1175-9. Crossref

32. Roy S, Vallepu S, Barrios C, Hunter K. Comparison of comorbid conditions between cancer survivors and age-matched patients without cancer. J Clin Med Res. 2018;10:911-9. Crossref

33. Schumacher M, Guennoun R, Stein DG, De Nicola AF. Progesterone: therapeutic opportunities for neuroprotection and myelin repair. Pharmacol Ther. 2007;116:77-106. Crossref

34. Roglio I, Bianchi R, Camozzi F, et al. Docetaxelinduced peripheral neuropathy: protective effects of dihydroprogesterone and progesterone in an experimental model. J Peripher Nerv Syst. 2009;14:36-44. Crossref

35. Beijers A, Mols F, Dercksen W, Driessen C, Vreugdenhil G. Chemotherapy-induced peripheral neuropathy and impact on quality of life 6 months after treatment with chemotherapy. J Community Support Oncol. 2016;12:401-6. Crossref

36. Zanville NR, Nudelman KN, Smith DJ, et al. Evaluating the impact of chemotherapy-induced peripheral neuropathy symptoms (CIPN-sx) on perceived ability to work in breast cancer survivors during the first year post-treatment. Support Care Cancer. 2016;24:4779-89. Crossref

37. Tofthagen C, Visovsky CM, Hopgood R. Chemotherapyinduced peripheral neuropathy: an algorithm to guide nursing management. Clin J Oncol Nurs. 2013;17:138-44. Crossref

38. Miaskowski C, Mastick J, Paul SM, et al. Chemotherapyinduced neuropathy in cancer survivors. J Pain Symptom Manage. 2017;54:204-18.e2. Crossref

(C) 2020 Advocate Aurora Health, Inc. 